\title{
CLASIFICAR ACCIONES. SOBRE LA CRÍTICA DE RAZ A LAS REGLAS CONSTITUTIVAS DE SEARLE
}

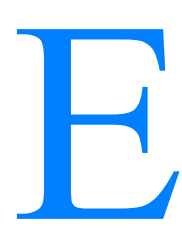

n su libro Razón práctica y normas, Joseph Raz critica la distinción trazada por Searle entre reglas regulativas y reglas constitutivas ${ }^{1}$. Esta distinción puede sintetizarse en las siguientes definiciones:

a) Las reglas regulativas regulan una conducta que puede ser descrita con independencia de la existencia de esa regla.

b) Las reglas constitutivas regulan una conducta que no podría ser descrita con independencia de esa regla y, por lo tanto, también constituyen esa conducta ${ }^{2}$.

En opinión de Raz, dada la caracterizacización que Searle hace de uno y otro tipo de normas, todas las reglas serían, simultáneamente, regulativas y constitutivas ${ }^{3}$. Creo que Raz tiene razón si su afirmación se entiende en el sentido de que todas las normas (también las regulativas o normas de conducta) tienen alguna dimensión constitutiva, pero disiento de él si su afirmación implica que no hay ninguna diferencia entre la «fuerza constitutiva» de las reglas regulativas y la de las reglas constitutivas. Me parece que esta diferencia existe, aunque probablemente no sea lo suficientemente marcada como para poder trazar una línea divisoria tajante entre los dos tipos de normas. Para detectar esta diferencia conviene relacionar la polémica acerca de las reglas constitutivas con algunas cuestiones de teoría de la acción y tratar de determinar cómo inciden uno y otro

${ }^{1}$ Joseph Raz, Razón práctica y normas, Centro de Estudios Constitucionales, Madrid, 1991, trad. de Juan Ruiz Manero, págs. 124-128.

${ }^{2}$ John Searle, Actos de habla, Ed. Cátedra, 1980, pág. 43, G. H. Von Wright, Norma y acción, Ed. Tecnos, Madrid, 1970, pág. 26.

${ }^{3}$ Joseph Raz, Razón práctica y normas, pág. 126. 
tipo de normas en nuestro proceso de individualización o descripción de acciones.

Comenzaré por resumir las consideraciones de Searle acerca de la distinción entre normas regulativas y normas constitutivas (1); expondré después la crítica de Raz (2) y, por último, trataré de reformular la distinción de forma que se aprecie de qué diferente manera «constituyen» acciones las reglas regulativas y las reglas constitutivas (3).

\section{Un mundo que se duplica. Las reglas constitutivas según Searle}

1.1. Searle no se ocupa de las reglas constitutivas con el genuino propósito de realizar una aportación a la teoría de las normas, sino con la intención de explicar ciertos interrogantes propios de la filosofía del lenguaje: «¿Cómo se relacionan las palabras con el mundo? ¿Cómo es posible que cuando un hablante está ante un oyente y emite una secuencia acústica ocurran cosas tan destacables como: el hablante quiere decir algo; el oyente comprende lo que se quiere decir; el hablante hace un enunciado, plantea una pregunta o da una orden? (...) ¿Cuál es la diferencia entre una sarta significativa de palabras y otra no significativa? $\gg^{4}$. La respuesta que propone Searle para este tipo de interrogantes parte de la hipótesis de que «hablar un lenguaje es tomar parte en una forma de conducta (altamente compleja) gobernada por reglas» ${ }^{5}$; aquí es donde entran en juego las reglas constitutivas y su distinción frente a las reglas regulativas. Un acto de habla tiene el significado que tiene porque existen ciertas reglas constitutivas que determinan que tenga tal significado.

«Las reglas constitutivas -escribe Searle- no regulan meramente: crean o definen nuevas formas de conducta (...). Las reglas regulativas regulan una actividad preexistente, una actividad cuya existencia es lógicamente independiente de las reglas. Las reglas constitutivas constituyen (y también regulan) una actividad cuya existencia es lógicamente dependiente de las reglas $\rangle^{6}$. La forma usual de las reglas regulativas es la de un imperativo: «Haz $\mathrm{X} »$ o « $\mathrm{Si} \mathrm{Y}$, haz $\mathrm{X}$ ». La forma usual de las reglas constitutivas sería «X cuenta como $\mathrm{Y} » \mathrm{O} \ll \mathrm{X}$ cuenta como $\mathrm{Y}$ en el contexto $\mathrm{C} »$.

${ }^{4}$ John Searle, Actos de habla, pág. 13.

${ }^{5}$ John Searle, Actos de habla, pág. 22.

${ }^{6}$ John Searle, Actos de habla, págs. 43. El subrayado es mío. La caracterización de las reglas constitutivas de Searle es semejante a la de von Wright. Von Wright y Searle coinciden también en sostener que las reglas constitutivas, además de definir nuevas formas de conducta, las regulan. 
Searle trata de aclarar su afirmación de que las reglas constitutivas crean nuevas formas de conducta. Esto -señala- podría entenderse en un sentido trivial, según el cual lo que estas reglas crean es la posibilidad de realizar una conducta conforme con ellas. En este sentido trivial, sin embargo, las reglas regulativas -y así lo reconoce Searle- también crearían formas nuevas de conducta. Por ejemplo, la regla según la cual se debe conducir por la derecha permite la acción de circular por la derecha conforme a la regla. Lo que Searle quiere decir cuando afirma que estas reglas crean la posibilidad de nuevas formas de conducta puede concretarse mejor teniendo en cuenta la distinción entre acción (en el sentido de una secuencia de movimientos corporales que da lugar a un cambio en el mundo) y descripción de la acción. Lo que las reglas constitutivas «crean» es la posibilidad de realizar acciones respecto de las cuales hay al menos una descripción que no sena posible si no existiera la regla. «Es posible -escribe Searle- que veintidós hombres puedan llevar a cabo los mismos movimientos físicos que realizan dos equipos en un partido de fútbol, pero si no hubiese reglas del fútbol, esto es, si no existiese con anterioridad el juego del fútbol, no habría ningún sentido en el que su conducta pudiese ser descrita como una instancia de jugar al fútbol» ${ }^{7}$.

Este carácter constitutivo está relacionado con otra peculiaridad que, según Searle, presentan también las reglas constitutivas: su condición «casi tautológica».

«Si nuestros paradigmas de reglas son reglas regulativas imperativas, tales reglas constitutivas no-imperativas probablemente nos sorprenderán como extremadamente curiosas, y difícilmente las reconoceremos incluso como reglas en absoluto. Obsérvese que tienen un carácter casi tautológico, pues lo que la «regla» parece ofrecer es parte de una definición de «jaque-mate» o de touchdown. El hecho de que, por ejemplo, se logre un jaque-mate en ajedrez de tal y tal manera puede aparecer bien como una regla, bien como una verdad analítica basada en el significado de «jaque-mate en ajedrez». El que tales enunciados puedan interpretarse como enunciados analíticos es una clave para el hecho de que la regla en cuestión es una regla constitutiva» ${ }^{8}$.

${ }^{7}$ John Searle, Actos de habla, pág. 45. En el mismo lugar, Searle admite que algunas reglas regulativas dan lugar a observaciones o comentarios acerca de conductas que no podrían realizarse si estas normas no existieran. Por ejemplo, decir «Alfredo fue muy descortés anoche» no tendría sentido sin ciertas normas (regulativas) de cortesía. Pero, en su opinión, lo que estas regulaciones crean no son realmente nuevas descripciones, sino valoraciones.

${ }^{8}$ John Searle, Actos de habla, pág. 43. El carácter analítico de las reglas constitutivas es lo que permite que algunos autores las vinculen a (o incluso identifiquen) 
A su vez, el carácter analítico de estas reglas tiene relación con la cuestión de su eficacia. Según Searle, no todas las reglas constitutivas tienen sanciones («¿Qué sanción tiene la regla de que el béisbol se juega con nueve hombres en cada equipo? ${ }^{9}$ ); aún más: en realidad, «ni siquiera es fácil ver cómo podría violarse la regla de lo que constituye el jaque mate en ajedrez, o el touchdown en fútbol americano» ${ }^{10}$. Cualquier desviación de las reglas de un juego no implica una violación de esas reglas, sino «salirse» del mismo, jugar a un juego distinto. Por lo tanto, en este sentido, la eficacia de las reglas constitutivas es «necesaria».

2.2. Algunos autores -por ejemplo, Guastini ${ }^{11}$-, entienden que la teoría de Searle crea una innecesaria "atmósfera metafísica», una atmósfera que nos conduce a un mundo que, mágicamente (a través del lenguaje), se «duplica». Searle, en efecto, asume en su teoría un compromiso ontológico que le lleva a admitir que en el mundo existen «hechos brutos» $\mathrm{y}$ «hechos institucionales». Son «hechos brutos» aquellos cuyo acontecer es independiente de las reglas, aunque pueden ser regulados por ellas, $\mathrm{y}$ «hechos institucionales» aquellos cuya existencia misma depende de las reglas constitutivas ${ }^{12}$. Pero este compromiso ontológico no viene asumido sólo por afirmar la existencia de reglas constitutivas junto a las reglas regulativas, sino por sostener además que los hechos institucionales no son reducibles a hechos brutos. «Cualquier periódico -escribe Searle- registra hechos del tipo siguiente: el señor Martínez ha contraído matrimonio con la señorita García; el Sporting ha vencido al Rayo por tres tantos a dos; a Pérez se le ha hallado convicto de robo; el Parlamento aprobó la investidura (...). No existe un conjunto simple de enunciados sobre propiedades físicas o psicológicas de estados de cosas al que sean reducibles los enunciados sobre hechos de este tipo» ${ }^{13}$. Y más adelante añade: «Las otras descripciones, las descripciones de los hechos brutos, pueden explicarse en términos de hechos institucionales. Pero los hechos institucionales pueden

las definiciones o reglas conceptuales (Eugenio Bulygin, «Sobre las normas de competencia», en Análisis lógico y Derecho, Centro de Estudios Constitucionales, Madrid, 1991, págs. 485-498).

${ }^{9}$ John Searle, Actos de habla, pág. 50.

${ }^{10}$ John Searle, Actos de habla, pág. 50.

${ }^{11}$ Ricardo Guastini, «Teoría de las reglas constitutivas: Searle, Ross, Carcaterra», en Alf Ross. Estudios en su homenaje, Revista de Ciencias Sociales, Universidad de Valparaíso, 1984, pág. 306.

${ }^{12}$ Sobre la distinción entre hechos brutos y hechos institucionales, véase John Searle, Actos de habla, págs. 58-61.

${ }^{13}$ John Searle, Actos de habla, pág. 59. 
explicarse solamente en términos de las reglas constitutivas subyacentes» ${ }^{14}$. Alf Ross también distingue entre estos dos tipos de reglas y entre actividades «naturales» e «instituciones», pero, como señala Guastini, «no sugiere en modo alguno que las actividades institucionales sean ontológicamente distintas de aquellas naturales. Las actividades institucionales son simplemente acciones a las cuales las reglas constitutivas confieren un significado; acciones que son «interpretadas» a través de reglas» ${ }^{15}$.

\section{Una crítica a las reglas constitutivas...}

La crítica de Raz a la distinción entre reglas regulativas y reglas constitutivas tiene como punto de partida el siguiente párrafo de Searle:

«Donde la regla es puramente regulativa, la conducta que está de acuerdo con la regla podría recibir la misma descripción o especificación (la misma respuesta a la pregunta «¿qué hizo él?») exista o no la regla, con tal de que la descripción o la especificación no haga referencia explícita a la regla. Pero allí donde la regla (o sistema de reglas) es constitutiva(o), la conducta que está de acuerdo con la regla puede recibir especificaciones o descripciones que no podría recibir si la regla no existiese» ${ }^{16}$.

La afirmación de que las reglas constitutivas crean nuevas formas de conducta debe entenderse -recordémoslo de nuevo- en el sentido de que lo que se crea es alguna clase nueva de acción o una nueva posibilidad de descripción de la conducta; esto es, debe entenderse que el cambio se produce en un nivel conceptual ${ }^{17}$. Esta facultad de las reglas constitutivas de crear nuevas formas de conducta es lo que, según Searle, distinguiría realmente las dos clases de normas. Estas no podrían diferenciarse sobre la base de que las normas regulativas

${ }^{14}$ John Searle, Actos de habla, pág. 61.

${ }^{15}$ Ricardo Guastini, «Teoría de las reglas constitutivas: Searle, Ross, Carcaterra», pág. 308.

${ }^{16}$ John Searle, Actos de habla, pág. 44.

${ }^{17}$ Conviene hacer aquí algunas estipulaciones terminológicas: Hablaré de «conducta» en el sentido de movimientos corporales. Asimilaré las expresiones «acción genérica» y «clases de acciones». A las acciones cuya existencia depende de reglas las llamaré acciones institucionales. A las acciones cuya existencia es independiente de reglas las llamaré acciones naturales. Forzando un poco las cosas, hablaré también de «descripciones naturales» y «descripciones institucionales», para referirme respectivamente a las descripciones de acciones naturales y a las descripciones de acciones institucionales. 
tienen una dimensión -la regulativa- que las reglas constitutivas no tienen, porque estas últimas también regulan la conducta que constituyen ${ }^{18}$.

Lo que Raz objeta a Searle es que, dada su caracterización de las reglas de uno y otro tipo, también las reglas regulativas tendrían una dimensión constitutiva y, por tanto, todas las reglas serían, simultáneamente, regulativas y constitutivas.

Supongamos -nos viene a decir Raz- que Mr. Jones es inspector de Hacienda, que debemos pagar $50 £$ de impuesto sobre la renta y que uno puede prometer algo diciendo «yo prometo». Tengamos en cuenta «la norma que impone el impuesto sobre la renta» y la «regla acerca del prometer» ${ }^{19}$. Analicemos después los siguientes pares de descripciones:

1. (a) «Dar $50 £$ a Mr. Jones».

(b) «Pagar el impuesto sobre la renta».

2. (a) «Decir 'yo prometo'».

(b) «Prometer».

Si no interpreto mal a Raz, su argumentación sería la siguiente: Tomemos la regla acerca del impuesto sobre la renta; ¿es una regla regulativa o una regla constitutiva? Según Raz, dada la caracterización de Searle de ambos tipos de reglas, se trataría tanto de una regla regulativa como de una regla constitutiva, porque podemos dar de la conducta conforme con ella tanto una descripción que es independiente de la existencia de la regla -1(a)- como una descripción que no sería posible sin la existencia de la regla -1(b)-. Lo mismo ocurre con la regla acerca del prometer: podemos dar de la conducta que está en conformidad con esta regla una descripción natural (independiente de la regla) -2(a)- o una descripción institucional (dependiente de la regla) -2(b)-. «Dado que para toda regla -concluye Raz- puede formularse un par semejante de descripciones de actos, todas las reglas son a la vez constitutivas y regulativas» ${ }^{20}$.

${ }^{18}$ John Searle, Actos de habla, pág. 43.

${ }^{19}$ Joseph Raz, Razón práctica y normas, pág. 125. Raz, por cierto, no aclara si se refiere a la norma que prescribe pagar el impuesto sobre la renta, o a la regla que define el impuesto sobre la renta, o si las considera una misma; ni si se refiere a la regla acerca de qué se debe hacer para realizar una promesa, o a la regla según la cual las promesas deben ser cumplidas, o si las considera una misma.

${ }^{20}$ Joseph Raz, Razón práctica y normas, pág. 126. Raz se adelante a una posible objeción: «Decir ‘yo prometo'»-sostendría el hipotético objetor- no basta para prometer algo; hay que decirlo con la intención de obligarse, de manera que la descripción correcta del acto sería «Decir "yo prometo' con la intención de crear una obligación voluntaria», y podría decirse que esta descripción presupone una regla acerca de las promesa, con lo que 2(a) (o su versión corregida) no sería una descripción natural. Raz contesta a esta posible objeción de dos maneras: En primer lugar, 


\section{3....y una réplica que no lo es tanto}

3.1. El párrafo de Searle -que he transcrito más arriba- en el que Raz basa su crítica es sumamente ambiguo. Entre las varias interpretaciones que me parece que pueden hacerse del mismo destacaré dos:

a) Primera interpretación: una regla es regulativa si la conducta realizada en conformidad con esa regla admite descripciones naturales y es constitutiva si la conducta realizada en conformidad con esa regla admite descripciones institucionales.

b) Segunda interpretación: una regla es regulativa si la conducta realizada en conformidad con esa regla sólo admite descripciones naturales y es constitutiva si, además, admite al menos una descripción institucional.

Las objeciones de Raz a Searle parecen indicar que Raz ha optado por la primera interpretación. Raz dice que todas las reglas serían regulativas y constitutivas, porque de la conducta en conformidad con cada una de ellas hay tanto descripciones naturales como descripciones institucionales. Si Raz optara por la segunda interpretación la conclusión de su argumentación sería que todas las reglas son constitutivas, porque de la conducta conforme con cada una de ellas se pueden dar descripciones naturales y, además, descripciones institucionales (ésta es la definición de regla constitutiva según la segunda interpretación). Pero lo que ocurre con esta interpretación no es menos grave para la distinción entre estos dos tipos de reglas: si todas las reglas son constitutivas, la distinción continúa careciendo de sentido.

Voy a proponer una tercera interpretación del párrafo de Searle:

c) Tercera interpretación: una regla es regulativa si la conducta realizada en conformidad con esa regla admite descripciones naturales y descripciones institucionales y es constitutiva si sólo admite descripciones institucionales.

Creo que esta interpretación sí que permite mantener -al menos en alguna medida, como se verá- la distinción entre reglas regulativas y reglas constitutivas.

la descripción revisada del acto no presupone la existencia de la regla acerca del prometer, sino que presupone meramente la creencia del agente en que hay una regla tal»; en segundo lugar, «precisamente por las mismas razones, dar a Mr. Jones $50 £$ no es un pago del impuesto sobre la renta salvo que se haga con la intención de pagar el impuesto sobre la renta». 
3.2. Recordemos algunas cuestiones básicas de una teoría de la acción ${ }^{21}$. Cuando observamos la conducta de los demás lo que en realidad estamos observando son secuencias de movimientos corporales que dan lugar a ciertos cambios en el mundo. Inmediatamente «clasificamos» esta «materia prima», de acuerdo con diversos criterios, como una acción de uno u otro tipo. Este proceso de clasificación es también un proceso de interpretación de la conducta, de atribución de un sentido a lo que vemos que el agente está haciendo. Si no entendemos que está haciendo el agente que observamos, si no podemos interpretar su conducta, no podremos clasificarla adecuadamente y la describiremos de una manera «no interpretada», probablemente en términos de movimientos corporales, aunque no necesariamente (podemos decir, por ejemplo: «está haciendo algo parecido a..., pero no es eso exactamente»). Los criterios que utilizamos para interpretar las acciones son muy variados: del lado de la acción nos fijamos en sus requisitos causales, la intención del agente o las consecuencias de lo que ha hecho; de otro lado, la «comunidad de vida», el lenguaje, las normas sociales, morales, etc., ofrecen clases de acciones («saludar», «vestirse», «cometer homicidio») en las que subsumir los movimientos corporales que observamos. Este proceso de clasificación o interpretación nos permite distinguir entre conducta no interpretada y conducta interpretada, o entre descripciones que no interpretan y descripciones que interpretan la conducta, pero se trata de una distinción gradual, en el sentido de que hay grados distintos de interpretación de la conducta: «correr» está más cerca de ser una descripción no interpretativa que «batir el récord de los cien metros lisos», pero «poner un pie delante del otro a un ritmo elevado» está aún más cerca ${ }^{22}$.

${ }^{21}$ Para una introducción a la teoría de la acción puede verse: Carlos S. Nino, Introducción a la filosofía de la acción humana, Eudeba, 1987; Jesús Mosterín, Racionalidad y acción humana, Alianza Universidad, 1987, capítulo 5 («La acción humana»); Carlos J. Moya, The Philosophy of Action. An Introduction, Polity Press, Cambridge, 1990; G. H. von Wright, Norma y acción, Ed. Tecnos, 1970, capítulo III («Acto y habilidad») y, del mismo autor, Explicación y comprensión, Alianza Universidad, 1980, capítulo 3 («Intencionalidad y explicación teleológica»).

${ }^{22}$ El límite, en el extremo de las descripciones no interpretativas, se encontraría en las acciones básicas: «Si acaso hay acciones -escribe Arthur Danto-, debe haber dos tipos distintos de acciones: las realizadas por un individuo $\mathrm{H}$, de quien puede decirse que las causó, y aquellas acciones, también realizadas por $\mathrm{H}$, de las que no puede decirse que él las causó. A estas últimas las designaré acciones básicas» (Arthur C. Danto, «Acciones básicas», Cuadernos de Crítica, 10, 1981, Instituto de Investigaciones Filosóficas, UNAM). En la teoría de von Wright una acción básica es aquella que el agente realiza «no por hacer alguna otra cosa, sino simple y directamente», esto es, aquellas «de las que no es verdad decir que se ejecutan mediante la realización de alguna otra cosa» (Von Wright, Explicación y comprensión, págs. 91 y 92). La acción de ventilar una habitación, por ejemplo, puede ser descrita como la acción de abrir una ventana, o como la acción de girar la 
Una descripción que interpreta una conducta es, pues, una descripción que la clasifica. «Flexionar un dedo sobre un gatillo» es una descripción no interpretativa (o con un nivel bajo de interpretación: no nos dice mucho acerca de lo que un agente ha hecho). Clasificar una conducta como un tipo de movimiento corporal siempre es posible, pero es una clasificación muy poco esclarecedora y poco interesante, en la mayoría de los casos. Esa misma conducta podría ser clasificada o descrita de manera más llamativa a medida que tengamos más datos para interpretarla mejor: los movimientos corporales «flexionar un dedo sobre un gatillo» podrían ser interpretados como gastar una broma, como dar un susto, como una amenaza, quizá se trate de una venganza, acaso un asesinato, quién sabe si un crimen pasional, un asesinato político o incluso el inicio de una nueva guerra mundial.

Tenemos entonces dos «clasificaciones» de las descripciones de la conducta: la que distingue entre «descripciones naturales» y «descripciones institucionales» (según que la descripción sea proporcionada o no por una regla) y la que distingue entre «descripciones interpretativas» $\mathrm{y}$ «descripciones no interpretativas». Podemos combinar ambas clasificaciones, con el siguiente resultado:

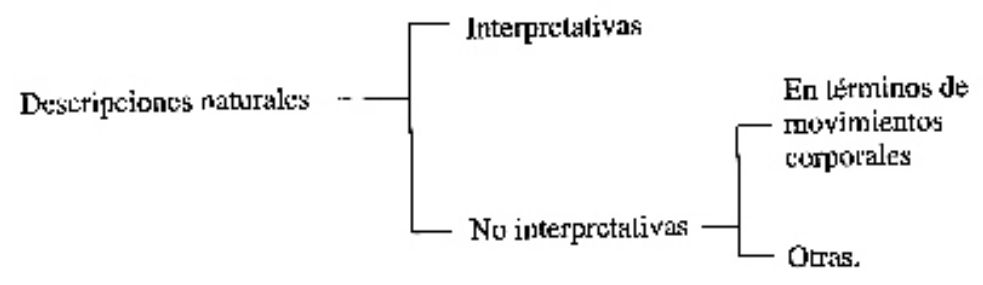

Descripcioncs institucionales —_ Intespretativas.

Las descripciones institucionales son siempre descripciones interpretativas (no podemos describir una secuencia de movimientos corporales como «jugar al ajedrez» sin haberlos interpretado). Las descripciones naturales, en cambio, pueden ser interpretativas («pedir auxilio») o no interpretativas («agitar los brazos»); las descripciones en términos de movimientos corporales son «descripciones naturales no interpretativas». Obsérvese que las descripciones en términos de movimientos corporales están siempre disponibles para cualquier conducta (salvo la exclusivamente mental, que no estamos considerando).

manija de la ventana y empujar de una determinada manera, o de realizar ciertos movimientos con nuestro cuerpo. Pero este proceso de «redescripción» de una acción ha de tener un final. Éste es, precisamente, la acción básica. 
3.3. Consideremos de nuevo los siguientes pares de descripciones:

1. (a) «Dar $50 £$ a Mr. Jones».

(b) «Pagar el impuesto sobre la renta».

2. (a) «Decir 'yo prometo'».

(b) «Prometer».

Podemos suponer que detrás de cada par de descripciones hay una misma secuencia de movimientos corporales. Designaré 1 a los movimientos corporales que hay tras las descripciones 1(a) y 1(b), y 2 a los movimientos corporales que hay tras las descripciones 2(a) y 2(b).

Supongamos que no existe la regla acerca de los impuestos. En este caso, 1(b) -que es una descripción institucional- no estaría disponible como descripción de 1. 1(a) -que es una descripción natural interpretativa-, en cambio, sí sería una descripción disponible para tales movimientos corporales. No se trataría de la descripción de un pago de impuestos, pero es una descripción que entendemos. Aun sin la regla del impuesto sobre la renta podemos clasificar la conducta y dar de ella una descripción interpretativa, aunque no institucional. Por último, también podríamos contentamos con describir la conducta de una manera no interpretada, en términos de movimientos corporales.

Vayamos ahora con el otro par de descripciones. Sin la existencia de la regla de las promesas, 2(b) -que es una descripción institucional- no estaría disponible como descripción 2. 2(b) no tendría sentido. ¿Lo tendría 2(a)? Creo que tampoco. Decir «yo prometo», si no existe la regla de las promesas, es como decir «yo...», donde los puntos suspensivos son una secuencia de sonidos sin sentido. Más exactamente, el único significado del enunciado «Decir 'yo prometo'», si no existe la regla de las promesas, es «Emitir los sonidos /yo prometo/», que es prácticamente una descripción en términos de movimientos corporales, o, al menos, una descripción en un «nivel bajo de interpretación». Sin la regla de las promesas no podemos comprender el sentido de esos movimientos corporales. Sin la regla de las promesas, en definitiva, ni 2(a) ni 2(b) son descripciones satisfactorias. En este caso, no tenemos ni una descripción institucional ni una descripción natural interpretativa para los movimientos corporales que he llamado «2».

Veamos otros ejemplos. La norma que establece una sanción en caso de homicidio se considera una norma de conducta. Sin ella no podríamos hablar de la acción de «cometer un homicidio» (descripción institucional), pero podríamos hablar de la acción de «matar» 
(descripción natural interpretativa). Aun sin la norma entenderíamos la conducta y tendríamos un buen arsenal conceptual para clasificarla. Además, podríamos dar de ella una descripción en términos de movimientos corporales. Vayamos ahora con una regla que se suela considerar constitutiva: por ejemplo, las reglas del ajedrez. ¿Qué ocurre con una descripción como «jugar al ajedrez»? Si queremos dar una descripción de esta actividad que sea totalmente independiente de las reglas del ajedrez tendremos que recurrir a descripciones en términos de movimientos corporales. No se me ocurre de qué otra manera podemos describir lo que vemos ${ }^{23}$.

Me parece que si seguimos analizando otros ejemplos nos encontraremos con dos tipos de conducta conforme a una regla: aquella que puede ser descrita de una manera interpretada exista o no la regla en cuestión ${ }^{24}$ y aquella que sólo puede ser descrita de una manera interpretada si existe la regla.

3.4. $\mathrm{Si}$ las anteriores consideraciones son acertadas, podríamos reformular la distinción entre reglas regulativas y reglas constitutivas de la manera siguiente:

Una regla es regulativa cuando la conducta conforme con ella puede ser descrita como una conducta no interpretada, como una acción natural interpretada o como una acción institucional. Una regla es constitutiva cuando la conducta conforme con ella sólo puede ser descrita como una conducta no interpretada o como una acción institucional ${ }^{25}$.

Aparentemente ésta es una caracterización extraña de ambos tipos de reglas, porque los dos tienen carácter constitutivo (los dos dan lugar a descripciones institucionales). Lo que ocurre con esta caracterización es que la distinción se convierte en una cuestión de grado: en cierto sentido, las reglas constitutivas tienen más «fuerza constitutiva» porque «crean» acciones que, en caso de no existir tales reglas, no podrían ser descritas de manera interpretada. Las

${ }^{23}$ Otros casos parecen estar a mitad de camino entre la regla de los impuestos y la de las promesas. Consideremos la norma del testamento válido. Si tratamos de describir la acción «hacer testamento válido» con independencia de la regla podemos dar una descripción que consista en una conjunción de distintas acciones, cada una de las cuales puede tener algún sentido para nosotros: escribir, expresar nuestra voluntad delante de dos testigos, firmar... Pero no tenemos -con independencia de la regla- una «etiqueta», un nombre para la «acción conjunta»: sin esa norma, la clasificación tendría «un nivel de interpretación muy bajo», haría referencia a una conjunción de acciones cuyo significado conjunto se nos escaparía.

${ }^{24}$ Por otra parte, si esta acción puede ser interpretada de otra manera aunque no exista la regla que estamos asumiendo es porque existen otras reglas o prácticas (sociales, morales... ) que configuran nuestros criterios de interpretación.

${ }^{25}$ Como ya hemos visto, una descripción no interpretativa siempre es posible. 
reglas regulativas también «crean» acciones, pero esas acciones podrían ser descritas de una manera interpretada si no existiera esa regla. Dicho de otra manera: las reglas regulativas «reclasifican» acciones ya clasificadas. Las reglas constitutivas «clasifican» la conducta no clasificada previamente (o clasificada con un nivel bajo de interpretación), por no existir -al margen de la regla- posibilidad de hacerlo. Searle y von Wright están en lo cierto cuando afirman que las reglas constitutivas regulan acciones que no existirían sin la regla, en el sentido de que sin la regla no cabría clasificar la acción de ninguna manera adecuada. Sin embargo, cuando afirman que las normas de conducta regulan acciones cuya existencia es independiente de la regla, aunque están señalando en la dirección adecuada, van más lejos de lo que debieran: están apuntando al hecho de que los movimientos corporales se podrían clasificar de otra manera, pero la clase de acción (la acción genérica) constituida por la regla no subsistiría, y por tanto no podría haber acciones individuales (descripciones de acciones) de ese tipo.

La distinción no sólo es una cuestión de grado en el sentido de que las reglas constitutivas tienen más fuerza constitutiva que las reglas regulativas. También lo es en el sentido de que hay -como hemos visto- grados distintos de «interpretación» de la conducta, lo que puede hacer difícil determinar si una determinada norma es regulativa o constitutiva.

Quizá esta reformulación pueda explicar por qué nuestras intuiciones (creo que no sólo la mía) apuntan hacia la existencia de «alguna» diferencia entre estas supuestas clases de normas (o entre el carácter constitutivo de unas y otras). Pero, por otra parte, el resultado de mi propuesta puede ser descorazonador para aquél en quien estas intuiciones sean especialmente fuertes, porque muestra que la distinción (al menos desde esta perspectiva) no es tajante.

DOXA-13 (1993) 